\title{
Intracellular delivery mechanism and brain delivery kinetics of biodegradable cationic bovine serum albumin-conjugated polymersomes
}

This article was published in the following Dove Press journal:

International Journal of Nanomedicine

5 July 2012

Number of times this article has been viewed

Zhiqing Pangl,2
Huile Gao ${ }^{1,2}$
Jun Chen
Shun Shen
Bo Zhang
Jinfeng Ren
Liangran Guo
Yong Qian ${ }^{1,2}$
Xinguo Jiang
Heng Mei,
'D
'Department of Pharmaceutics,
School of Pharmacy, ${ }^{2}$ Key Laboratory
of Smart Drug Delivery, Ministry of
Education and PLA, Fudan University,
Shanghai, ${ }^{3}$ Institute of Hematology,
Union Hospital, Tongji Medical
College, Huazhong University of
Science and Technology, Wuhan, Hubei,
People's Republic of China

Correspondence: Xinguo Jiang Department of Pharmaceutics, School of Pharmacy, Fudan University, Zhangjiang Campus, 826 Zhangheng

Road, Shanghai 201203,

People's Republic of China

$\mathrm{Tel}+862 \quad 151980067$

Fax +862151980069

Email xgjiang@shmu.edu.cn

Heng Mei

Institute of Hematology, Union Hospital,

Tongji Medical College, Huazhong

University of Science and Technology,

Wuhan, Hubei 430022, PR China

Tel +862785726007

Fax +862 785726387

Email mayheng@I26.com
Background: A novel brain drug delivery system using cationic bovine serum albumin (CBSA)-conjugated biodegradable polymersomes (CBSA-PO) was prepared, and its intracellular delivery mechanism and brain delivery kinetics were evaluated.

Methods and results: Biodegradable poly(ethylene glycol)-poly( $\varepsilon$-caprolactone) (PEG-PCL) was used to prepare the polymersomes, and thiolated CBSA was conjugated with the surface of the polymersome. Transmission electron microscopy and dynamic light scattering showed that the CBSA-PO had a round and vesicle-like shape, with a mean diameter of around $100 \mathrm{~nm}$. Coupling of CBSA with polymersomes was confirmed by X-ray photoelectron spectroscopy. Uptake of CBSA-PO by bEnd. 3 cells was significantly higher than that of unconjugated polymersomes, but was inhibited by low temperature, free CBSA, and poly-L-lysine, indicating that endocytosis was energy-driven and absorptive-mediated. Cell viability assays confirmed the good safety profile of biodegradable CBSA-PO. Pharmacokinetic results demonstrated that the polymersomes had long circulation times, and CBSA conjugation on the polymersomes significantly increased the blood-brain barrier permeability surface area product by 3.6-fold and the percentage of injected dose per gram brain (\% ID/g brain) by 2.1 -fold. Capillary depletion experiments showed that CBSA-PO was distributed into the brain parenchyma in a time-dependent manner, with few polymersomes detected, indicating that conjugation of polymersomes with CBSA significantly improved their transcytosis across the brain-blood barrier.

Conclusion: These results suggest that CBSA-PO is a promising drug brain delivery carrier with low toxicity.

Keywords: brain delivery kinetics, biodegradable polymersomes, cationic albumin, brain-blood barrier

\section{Introduction}

The delivery of therapeutic agents to treat diseases of the brain is far from efficient due to the blood-brain barrier, which separates blood from the underlying brain cells, protects neurons, and preserves homeostasis in the central nervous system. ${ }^{1,2}$ It has been shown that nearly $100 \%$ of large-molecule drugs and $>98 \%$ of small-molecule drugs do not cross the blood-brain barrier. Consequently, the prognosis for many neurological disorders remains poor, and targeting drug delivery to the brain is a therapeutic challenge. A great deal of effort is presently focused on developing strategies to deliver active drugs to the brain effectively. Recently, nanocarrier systems, ${ }^{3}$ such as micelles, liposomes, nanoparticles, and dendrimers, ${ }^{4}$ have been under investigation as potential agents for drug delivery to the brain. Like most drugs, nanocarriers show low permeability at the blood-brain barrier. Thus, targeting ligands, such as transferrin, ${ }^{5}$ 
angiopep peptide, ${ }^{4}$ apolipoprotein,${ }^{6}$ rabies virus glycoprotein peptide, ${ }^{7}$ and phage display peptide, ${ }^{8}$ have been used to modify the surface of nanocarriers to increase the efficiency of uptake by the brain. However, there remain issues of uptake efficiency, especially in the brain parenchyma. Therefore, novel nanocarriers rationally functionalized with targeting ligands are still necessary for the construction of effective brain-targeted delivery systems.

Polymersomes are a new class of vesicles self-assembled from amphiphilic block copolymers, which are currently attracting great interest because of their structural similarity to living organelles and potential applications as nanosized reactors or drug delivery systems. ${ }^{9-12}$ The last decade has shown polymersomes to be promising alternatives to liposomes, due to their remarkable, attractive, and feasible characteristics, including stealthiness, improved stability, and ease of functionalization. ${ }^{12-14}$ In addition, desirable and intelligent polymersomes can be designed to combine multiple features required for drug delivery, such as biodegradability, ${ }^{15}$ targeting capability, ${ }^{16}$ and responsiveness to biologically relevant stimuli, ${ }^{17}$ such as $\mathrm{pH}$, temperature, light, and reductive environments. Therefore, polymersomes are good candidates for drug delivery, and are currently being developed by many research groups. However, the vast majority of present efforts are focused on fabrication of novel functional polymersomes, whereas in vivo exploitation of biodegradable polymersomes as brain drug delivery systems has not often been investigated.

Our group has reported a novel brain delivery system using poly(ethylene) glycol-poly( $\varepsilon$-caprolactone) (PEG-PCL) polymersomes conjugated with the mouse-antirat monoclonal antibody, OX26, ${ }^{18}$ which can undergo endogenous receptor-mediated transcytosis across the blood-brain barrier. However, as a surface-conjugated targeting agent, OX26 has many disadvantages, including an inability to bind to the human transferrin receptor, as well as the difficulty in preparing humanized antibodies and the potential risk of disturbing iron homeostasis in the brain, ${ }^{19}$ which greatly limit the application of OX26 in drug delivery to the brain. Cationic bovine serum albumin (CBSA) has been shown to have a greater binding capacity for brain microvessels and an increased accumulation profile in the brain via an absorptivemediated transcytosis pathway, indicating that CBSA might be a potential brain targeting vector despite its nonspecificity and immunogenicity. ${ }^{20,21}$ In our previous work, PEG-polyL-lactide nanoparticles conjugated with CBSA proved to be promising brain delivery carriers for small-molecule and large-molecule drugs, including peptides and genes..$^{22,23}$
In addition, CBSA was easier to prepare than the monoclonal antibody. Having these properties, CBSA was identified to be a promising alternative brain targeting vector.

Although many researchers have reported increased brain delivery of nanocarriers functionalized with targeting ligands, little is known about nanocarrier distribution in the brain parenchyma after transport across the blood-brain barrier. Nanocarrier content in the total brain includes nanocarriers that cross the blood-brain barrier and those that bind to or are endocytosed by endothelial cells without transcytosis into the post-capillary compartment. Identification of nanocarriers in the brain parenchyma and cerebral endothelium could provide direct evidence of nanocarrier transport across the blood-brain barrier and an indepth insight into the kinetics of the process. Therefore, the present study evaluated the brain delivery kinetics of a polymersome-based molecular "Trojan horse", whereby CBSA was conjugated to the surfaces of polymersomes (CBSA-PO). Biodegradable PEG-PCL was used to prepare the polymersomes by nanoprecipitation. The morphology, particle size, and surface characteristics of CBSA-PO were characterized by transmission electron microscopy, dynamic light scattering, and x-ray photoelectron spectroscopy. The cellular uptake and pharmacokinetics of CBSA-PO were investigated using coumarin-6 as a fluorescent probe, and the brain delivery kinetics of CBSA-PO were characterized using a well established capillary depletion technique. ${ }^{24,25}$ Cell viability assays was performed to confirm the safety and biodegradability of CBSA-PO.

\section{Materials and methods Materials and animals}

Two diblock copolymers, MPEG3K-PCL15.8K and maleimide-PEG3.4K-PCL16K, were synthesized by ring-opening polymerization of $\varepsilon$-CL, using MPEG (molecular weight $3000 \mathrm{Da}$, NOF Corporation, Tokyo, Japan) and maleimide-PEG (molecular weight $3400 \mathrm{Da}$, Nektar Therapeutics, San Francisco, CA), as described elsewhere. ${ }^{18}$ Traut's reagent (2-iminothiolane) was purchased from Sigma-Aldrich (Saint Louis, MO). Coumarin-6 and coumarin-7 were obtained from Sigma-Aldrich, and Ellmann's reagent (5,5-dithiobis-2-nitrobenzoic acid) was obtained from Acros Organics (Geel, Belgium). Bovine serum albumin (BP0042, Roche Applied Science, Basel, Switzerland) was used to prepare the CBSA, as in our previous work. ${ }^{26}$ Isoelectric focusing of CBSA showed that the pI of CBSA was about 8.5 , and sodium dodecyl sulfate polyacrylamide gel electrophoresis determined that the molecular weight of CBSA was approximately $66 \mathrm{kDa}$. 
A bEnd.3 cell line was obtained from the American Type Culture Collection (Rockville, MD). Fetal bovine serum and a related cell culture medium were purchased from Invitrogen (Gibco, Carlsbad, CA). Plastic cell culture dishes, plates, and flasks were ordered from Corning Incorporation (Lowell, MA). Double-distilled water was purified using a Millipore Simplicity System (Millipore, Bedford, MA). All other chemicals were of analytical reagent grade and were used without further purification.

Sprague-Dawley rats weighing 200-230 g were obtained from the Shanghai SLAC Laboratory Animal Co, Ltd (Shanghai, China). The animals used for the experiments were treated according to protocols evaluated and approved by the ethics committee of Fudan University.

\section{Preparation of cationic albumin- conjugated polymersomes \\ Biodegradable polymersomes conjugated with cationic albumin}

Biodegradable polymersomes were made using a blend of MPEG-PCL and maleimide-PEG-PCL by nanoprecipitation. ${ }^{11}$ Briefly, $20 \mathrm{mg}$ of MPEG-PCL and $2 \mathrm{mg}$ of maleimide-PEGPCL were dissolved in $1.0 \mathrm{~mL}$ of tetrahydrofuran. The polymer solution was then injected slowly into $10 \mathrm{~mL}$ of $0.01 \mathrm{M}$ phosphate-buffered saline ( $\mathrm{pH}$ 7.4). Mild stirring was performed to induce self-assembly. After 30 minutes, the solution was dialyzed against $0.01 \mathrm{M}$ phosphate-buffered saline (pH 7.4) to remove the tetrahydrofuran. The polymersome solutions were concentrated using TM Ultra-4 (Amicon, Billerica, MA) concentrator tubes with ultrafilter membranes (molecular weight cut-off, $10 \mathrm{kDa}$ ). CBSA was thiolated and characterized as in our previous work. ${ }^{27}$ Thiolated CBSA was then incubated with the concentrated polymersomes with a thiol group to maleimide group molar ratio of 1:3 for 8 hours. The resulting CBSA-PO was purified by passage through a Sepharose CL-4B column eluted with $0.01 \mathrm{M}$ phosphatebuffered saline ( $\mathrm{pH}$ 7.4). Preparation of the polymersomes loaded with coumarin- 6 was the same as that used for the blank CBSA-PO, except that approximately $120 \mu \mathrm{g}$ of coumarin-6 was added to the copolymer solution.

\section{Morphology, particle size, and surface analysis}

The shape and size of the unloaded polymersomes were observed by transmission electron microscopy (JEM-1230, JEOL, Tokyo, Japan) following negative staining with $1 \%$ uranyl acetate solution. The mean diameter and zeta potential of the CBSA-PO were determined by dynamic light scattering using a zeta potential/particle sizer (Nicomp ${ }^{\text {TM }} 380$ ZLS,
Particle Sizing Systems, Santa Barbara, CA). Surface CBSA densities were determined using the bicinchoninic acid protein assay combined with turbidimetry, as previously described. ${ }^{18}$ The surface chemical composition of the polymersomes, CBSA-PO, and a mixture of maleimidePEG-PCL:MPEG-PCL (1:10) were analyzed by x-ray photoelectron spectroscopy on an RBD-upgraded PHI5000C ESCA system (Perkin Elmer, Boston, MA) as in our previous work. ${ }^{27}$

\section{Drug-loading efficiency and in vitro release of coumarin- 6 from CBSA-PO}

The drug-loading efficiency of coumarin-6 in CBSA-PO was determined by high-performance liquid chromatography combined with turbidimetry. ${ }^{27}$ In vitro release of coumarin-6 from CBSA-PO was performed in phosphate-buffered saline ( $\mathrm{pH} 4$ or 7.4 ) and in rat plasma. ${ }^{18}$ The samples released were analyzed by high-performance liquid chromatography (Agilent 1200, Santa Clara, CA) with a fluorescence detector at an excitation wavelength of $465 \mathrm{~nm}$ and an emission wavelength of $502 \mathrm{~nm} .{ }^{27}$ Samples were shielded from light throughout the experimental procedure.

\section{Quantitative determination of cell uptake by coumarin-6 loaded polymersomes}

The bEnd. 3 cells were seeded onto a 24 -well plate at a density of $10^{5}$ per well and incubated at $37^{\circ} \mathrm{C}$ for 24 hours. After a 30-minute incubation in Dulbecco's modified Eagle's medium (DMEM), the cells were treated with $100 \mu \mathrm{g} / \mathrm{mL}$ of coumarin-6-loaded CBSA-PO or polymersomes and various inhibitors for 60 minutes, ie, DMEM (control), CBSA (1 mg/mL), poly-L-lysine $(500 \mu \mathrm{g} / \mathrm{mL}), 0.1 \%$ (w/w) sodium azide, $450 \mathrm{mmol} / \mathrm{L}$ sucrose, $20 \mu \mathrm{mol} / \mathrm{L}$ phenylarsine oxide (PheASO), or $10 \mu \mathrm{g} / \mathrm{mL}$ filipin. After washing with ice-cold phosphate-buffered saline and acid buffer (120 mmol/L NaCl, $20 \mathrm{mmol} / \mathrm{L}$ sodium barbital, $20 \mathrm{mmol} / \mathrm{L}$ sodium acetate $[\mathrm{pH} 3])$ at $4^{\circ} \mathrm{C}$ for 5 minutes, the cells were solubilized with $0.4 \mathrm{~mL}$ of $1 \%$ Triton X-100 at $4^{\circ} \mathrm{C}$ overnight. Afterwards, $25 \mu \mathrm{L}$ of the cell lysate from each well was sampled to determine the total cell protein content using a bicinchoninic acid protein assay kit. The rest of the cell lysate was used for extraction and high-performance liquid chromatography determination of coumarin- $6 .{ }^{27}$ A standard curve was constructed for the polymersomes by suspending them at different concentrations $(5-200 \mu \mathrm{g} / \mathrm{mL})$ in $1 \%$ Triton $\mathrm{X}-100$, followed by the same extraction and high-performance liquid chromatography determination procedure as that used for the samples. Polymersome uptake 
is expressed as percent uptake of the control. To evaluate the effect of temperature on cell uptake, the cells were treated according to the same procedure as described above, except that the cells were incubated for one hour with $100 \mu \mathrm{g} / \mathrm{mL}$ coumarin-6-loaded CBSA-PO or polymersomes at $4{ }^{\circ} \mathrm{C}$ and $37^{\circ} \mathrm{C}$, respectively. The uptake index was expressed as polymersomes $(\mu \mathrm{g}) /$ cellular protein $(\mathrm{mg})$.

\section{In vitro cytotoxicity of CBSA-PO}

The bEnd. 3 cells were seeded into a 96-well plate at a density of $10^{4}$ per well and incubated for 24 hours before exposure to CBSA, polymersomes, and CBSA-PO samples in DMEM, in a series of concentrations for an additional 4 hours at $37^{\circ} \mathrm{C}$. Eight wells treated only with DMEM were prepared as controls. After exposure, the cytotoxicity of these formulations was assayed using the MTT method. ${ }^{28}$ These experiments were performed in quadruplicate.

\section{Pharmacokinetics and brain delivery kinetics of CBSA-PO \\ Pharmacokinetic experiments}

Pharmacokinetic experiments were performed as previously described. ${ }^{18}$ Briefly, rats were anesthetized intraperitoneally with $400 \mathrm{mg}$ of chloral hydrate per $\mathrm{kg}$ of body weight. Coumarin-6-labeled CBSA-PO, polymersomes, or CBSA-PO plus free CBSA (10 mg/kg) was injected into the left femoral vein at a dose of $10 \mathrm{mg} / \mathrm{kg}$. Blood samples were collected via a cannula implanted in the left femoral artery at $0.25,1,2,5$, $15,30,60$, and 120 minutes following intravenous injection. The blood volume was replaced with an equal volume of saline. At 120 minutes, the animals were decapitated, and the brain and other major organs, including the liver, spleen, heart, lung, and kidney, were collected, followed by a rapid wash with cold saline to remove any superficial blood. An additional incision was made into the heart to squeeze any remaining blood out of the ventricles.

\section{Capillary depletion}

The transport kinetics of CBSA-PO across the blood-brain barrier in vivo were investigated using a capillary depletion technique, as described elsewhere. ${ }^{24,25}$ This technique separates the brain homogenate into the vascular pellet compartment and the postvascular supernatant compartment, which represents polymersomes bound to or endocytosed into endothelial cells and those transcytosed into the brain interstitial space, respectively. In brief, Sprague Dawley rats were randomized into three groups and administered coumarin-6-labeled CBSA-PO, polymersomes, or CBSA-
PO plus free CBSA $(10 \mathrm{mg} / \mathrm{kg})$ at a polymersome dose of $10 \mathrm{mg} / \mathrm{kg}$ via the tail vein. At hours $0.5,1,2$, and 4 , the rats were decapitated and blood samples were collected. Subsequently, the brains were rapidly removed, weighed, and homogenized in $7 \mathrm{~mL}$ of cold $13 \%$ dextran solution. After centrifugation at $3000 \mathrm{~g}$ for 15 minutes at $4^{\circ} \mathrm{C}$, a $5 \mathrm{~mL}$ aliquot of the homogenate was separated into the postvascular supernatant and the vascular pellet. Both fractions, as well as the whole homogenate, were carefully sampled for extraction and high-performance liquid chromatography determination of coumarin- 6 as described below.

\section{Analytical procedure}

The tissue samples were homogenized on ice with three-fold volumes of deionized water, followed by addition of $10 \mu \mathrm{L}$ of 7-coumarin (internal standard, $50 \mathrm{ng} / \mathrm{mL}$ ). The samples were extracted with $2 \mathrm{~mL}$ of $\mathrm{n}$-hexane after 2 minutes of intense vortexing. After centrifugation at 12,000 rpm for 10 minutes, the supernatant was transferred and evaporated under a gentle stream of nitrogen at $40^{\circ} \mathrm{C}$. The residue was dissolved in $500 \mu \mathrm{L}$ of mobile phase, and $20 \mu \mathrm{L}$ was injected into the high-performance liquid chromatography system. The blood plasma samples, postvascular supernatants $(0.5 \mathrm{~mL})$, vascular pellets $(0.2 \mathrm{~g})$, and whole brain homogenate $(0.5 \mathrm{~mL})$ were treated in the same manner, without addition of water.

The chromatographic analysis was performed using a high-performance liquid chromatography system (Shimadzu Scientific Instruments Inc, Tokyo, Japan), including a pump (Model LC-10AT) and a fluorescence detector (Model RF-10AXL; $\left.\lambda_{\text {ex }}=465 \mathrm{~nm}, \lambda_{\mathrm{em}}=502 \mathrm{~nm}\right)$. Using methanol/ $\mathrm{H}_{2} \mathrm{O}(96: 4, \mathrm{v} / \mathrm{v})$ as the mobile phase, at a flow rate of $1.2 \mathrm{~mL}$ per minute, chromatographic separations were achieved using a $\mathrm{C}_{18}$ analytical column $(5 \mu \mathrm{m}, 4.6 \times 200 \mathrm{~mm}$, Dikma Diamonsil RP18 column) equipped with a guard column (Nova-Pak, Waters, Milford, MA) at $35^{\circ} \mathrm{C}$.

\section{Data analysis}

The concentration of polymersomes in blood and tissues (including the homogenate, vascular pellet, and postvascular supernatant) were expressed as \% ID $/ \mathrm{mL}$ (\% ID, percentage of injected dose) or $\%$ ID $/ g$. Using nonlinear regression, the plasma data were fitted to a biexponential equation, $A(t)=A_{1} e^{-k_{1} t}+A_{2} e^{-k_{2} t}$, where $A(t)$ is $\%$ ID per $\mathrm{mL}$ of plasma. ${ }^{29}$ Pharmacokinetic parameters, such as plasma clearance and steady-state volume of distribution $\left(V_{\mathrm{ss}}\right)$, were calculated from $A_{1}, A_{2}, k_{1}$, and $k_{2}$. Area under the blood concentration curve $\left(\mathrm{AUC}_{0-\mathrm{t}}\right)$ was calculated by noncompartmental data analysis of blood concentra- 
tions. The volume of distribution $\left(V_{\mathrm{d}}\right)$ in the brain after intravenous injection was determined from the ratio of the amount of drug in brain tissue (\% ID per g tissue) divided by the concentration of drug $(\% \mathrm{ID} / \mathrm{mL})$ in terminal plasma. The brain permeability surface area (PS) product was determined as follows: ${ }^{30}$

$$
P S=\frac{\left[V_{d}-V_{0}\right] C p(T)}{\int_{0}^{t} C p(t) d t}
$$

where $C p(T)$ was the terminal blood concentration and $V_{0}$ was the brain polymersome distribution volume. According to the method reported by Huwyler et al and $\mathrm{Lu}$ et al, ${ }^{30,31}$ $V_{0}$ was determined as the amount of polymersomes in brain tissue (\% ID per g tissue) 2 minutes after intravenous injection divided by the plasma concentration of polymersomes $(\% \mathrm{ID} / \mathrm{mL})$ at the same time point as that described previously. ${ }^{18}$ Brain uptake was expressed as \% ID per $g$ of tissue and calculated as: $\% I D / g(t)=P S \times A U C_{0-t}$.

\section{Results}

\section{Characterization of cationic albumin- conjugated polymersomes}

\section{Morphology, particle size, and zeta potential}

Transmission electron microscopy (Figure 1) showed that CBSA-PO spontaneously assembled by a blend of MPEG3K-PCL7.6K and maleimide-PEG3.4K-PCL7K had a relatively narrow size distribution and that conjugation with CBSA did not change their vesicle-like shape. Dynamic light scattering showed that the average diameter of the polymersomes was around $95 \mathrm{~nm}$ (Table 1). A slight

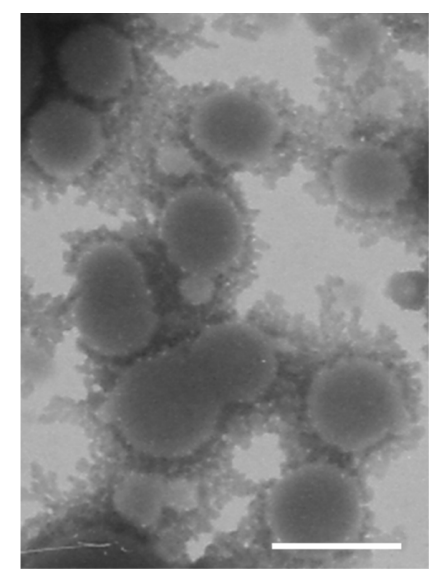

Figure I Transmission electron microscopy images of cationic albumin-conjugated biodegradable polymersomes negatively stained with I\% uranyl acetate solution (bar $100 \mathrm{~nm}$ ).
Table I Particle sizes and zeta potential values of $\mathrm{PO}$ and CBSA-PO, loaded or not loaded with coumarin-6 $(n=3)$

\begin{tabular}{lcc}
\hline Vesicle & $\begin{array}{l}\text { Mean size, } \\
\mathbf{n m}\end{array}$ & \multicolumn{1}{c}{$\begin{array}{l}\text { Zeta potential } \\
(\mathbf{m V})^{\mathbf{a}}\end{array}$} \\
\hline PO & $95.2 \pm 4.3$ & $-19.2 \pm 0.8$ \\
CBSA-PO & $101.1 \pm 7.6$ & $9.3 \pm 0.5$ \\
Coumarin-6-loaded PO & $96.6 \pm 5.2$ & $-20.3 \pm 0.7$ \\
Coumarin-6-loaded CBSA-PO & $102.7 \pm 5.5$ & $9.5 \pm 0.3$ \\
\hline
\end{tabular}

Note: a Measured in $\mathrm{NaCl}$ solution $\left(10^{-3} \mathrm{M}\right)$.

Abbreviations: $\mathrm{PO}$, polymersomes; CBSA, cationic bovine serum albumin.

increase in vesicle size was observed for CBSA-PO, which had an average diameter of around $101 \mathrm{~nm}$. There was no significant difference in vesicle size between unloaded CBSA-PO and CBSA-PO loaded with coumarin-6, indicating that the incorporation of coumarin- 6 did not influence vesicle size.

The Zeta potential values for polymersomes, CBSA-PO, and coumarin-6-loaded CBSA-PO in $0.001 \mathrm{M} \mathrm{NaCl}$ solution (pH 7.0) are shown in Table 1. Compared with the polymersomes, the zeta potential value of CBSA-PO increased dramatically, from $-20 \mathrm{mV}$ to around $+9 \mathrm{mV}$, indicating that CBSA conjugation caused inversion of the zeta potential. No significant difference in zeta potential was observed between CBSA-PO loaded with coumarin-6 and unloaded CBSA-PO, indicating that incorporation of coumarin- 6 did not influence the zeta potential.

\section{Surface analysis}

The mean number of CBSA molecules per polymersome on the surface of the CBSA-PO was $95.8 \pm 9.2$, which has been shown in other studies to be a reasonable density for brain targeting. ${ }^{31}$ Quantitative data for the surface chemical composition of maleimide-PEG-PCL:MPEG-PCL $(1: 10)$ copolymers, polymersomes, and CBSA-PO were provided by the X-ray photoelectron spectroscopy analysis (Table 2). Surface nitrogen was only detected in the CBSA-PO sample, and the existence of CBSA incorporated onto the polymersome surface was determined from the N1s x-ray photoelectron spectrum (Figure 2D). Decomposition of the $\mathrm{C} 1 \mathrm{~s}$ envelope revealed the presence of four types of carbon (Figure 2). Their relative composition ratios, based on area, are shown in Table 2. Because peak 2, at a binding energy of $286.6 \mathrm{eV}$, was regarded as an indicator of MPEG, ${ }^{32}$ the presence of a PEG corona layer on the polymersome surface could be confirmed by the increased $\mathrm{C}-\mathrm{O}-\mathrm{C}$ peak ratio of $30.8 \%$ for polymersomes and $33.6 \%$ for CBSA-PO, in comparison with $18.7 \%$ for the maleimide-PEG-PCL:MPEGPCL copolymer mixture. 
Table 2 X-ray photoelectron spectroscopy of maleimide-PEG-PCL:MPEG-PCL mixture (I:I0), PO-coupled with or without CBSA

\begin{tabular}{|c|c|c|c|c|c|c|c|c|c|}
\hline \multirow[t]{5}{*}{ Samples } & \multicolumn{3}{|c|}{ XPS elemental ratio (\%) } & \multicolumn{4}{|c|}{ XPS CIs envelope ratios (\%) } & \multirow{2}{*}{\multicolumn{2}{|c|}{$\begin{array}{l}\text { XPS OIs envelope } \\
\text { ratios (\%) }\end{array}$}} \\
\hline & \multirow[t]{4}{*}{ C } & \multirow[t]{4}{*}{0} & \multirow[t]{4}{*}{$\mathbf{N}$} & \multirow[t]{2}{*}{$\mathrm{C}-\mathrm{C} / \mathrm{C}-\mathrm{H}$} & \multirow[t]{2}{*}{ C-O-C } & \multirow[t]{2}{*}{$\mathrm{C}-\mathrm{O}-\mathrm{C}=\mathrm{O}$} & \multirow[t]{2}{*}{ O-C $=0$} & & \\
\hline & & & & & & & & $\mathrm{O}=\mathrm{C}$ & O-C \\
\hline & & & & \multicolumn{6}{|c|}{ Binding energy $(\mathrm{eV})$} \\
\hline & & & & 285.0 & 286.6 & 287.3 & 289.4 & 533.5 & 534.4 \\
\hline Copolymers & 75.1 & 24.9 & $*$ & 7.5 & 18.7 & 56.6 & 17.2 & 34.7 & 65.3 \\
\hline PO & 74.2 & 25.8 & $*$ & 27.4 & 30.8 & 27.3 & 14.5 & 44 & 56 \\
\hline CBSA-PO & 74.1 & 25.5 & 0.4 & 13.5 & 33.6 & 33.1 & 19.8 & 37.7 & 62.3 \\
\hline
\end{tabular}

Notes: *Below the detection limit. Copolymers are maleimide-PEG-PCL:MPEG-PCL mixture (I:10).

Abbreviations: XPS, x-ray photoelectron spectroscopy; PO, polymersomes; PEG, poly(ethylene glycol); PCL, poly( $(\varepsilon$-caprolactone); CBSA, cationic bovine serum albumin; MPEG, methoxy poly(ethylene glycol).

\section{Drug-loading efficiency and in vitro release of coumarin-6 from CBSA-PO}

The drug-loading efficiency of coumarin-6 loaded CBSA-PO was $0.8 \%$, which is high enough for qualitative and quantitative study in vitro and in vivo. ${ }^{31,33}$ In vitro release tests showed that, after a 48-hour incubation period, approximately $0.50 \%, 0.39 \%$, and $2.58 \%$ of coumarin- 6 was released from CBSA-PO in $\mathrm{pH} 4.0$ phosphate-buffered saline, $\mathrm{pH} 7.4$ phosphate-buffered saline, and rat plasma, respectively. This lack of release indicates that the fluorescence signal detected in the blood and organ samples could be attributed mainly to the coumarin- 6 encapsulated within the polymersomes and that coumarin- 6 could be an accurate probe for polymersome behavior in vivo.

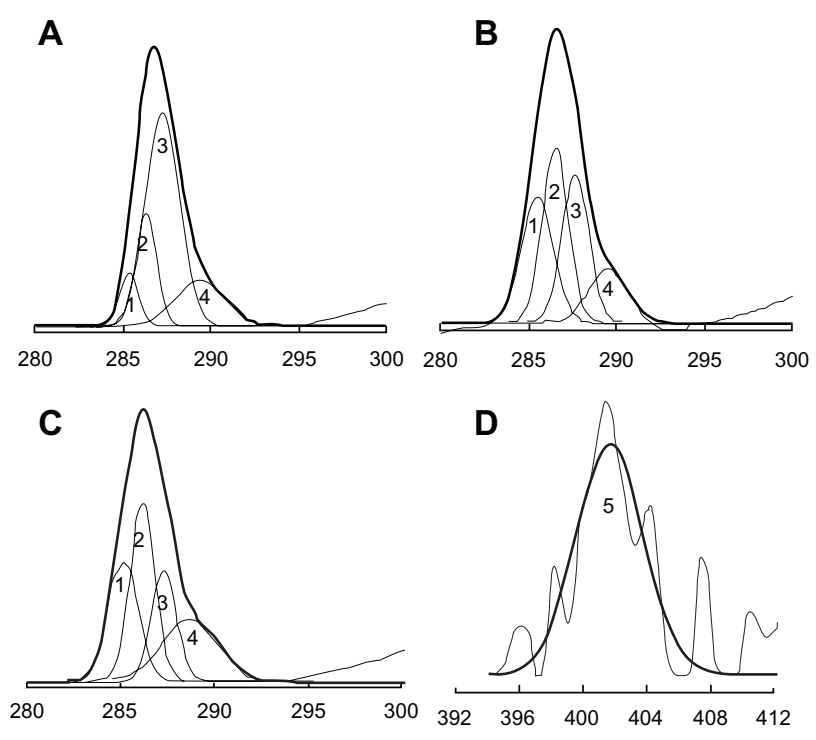

Figure 2 Carbon $\mathrm{Cl}$ s envelopes from $\mathrm{X}$-ray photoelectron spectroscopy analysis of (A) mixture of maleimide-PEG-PCL:MPEG-PCL (I:I0) copolymers, (B) PO without CBSA attached, and (C) CBSA-PO (maleimide-PEG-PCL:MPEG-PCL, 1:10 ratio). (D) Nitrogen NIs envelopes from x-ray photoelectron spectroscopic analysis of CBSA-PO.

Abbreviations: PO, polymersomes; PEG, poly(ethylene glycol); CBSA, cationic bovine serum albumin; PCL, poly( $\varepsilon$-caprolactone); MPEG, methoxy poly(ethylene glycol).

\section{Cellular uptake of polymersomes}

bEnd. 3 cells are an immortalized mouse brain endothelial cell line with specific endothelial properties, blood-brain barrier characteristics, and amenability to numerous molecular interventions. These cells were chosen as a simple blood-brain barrier model to study the intracellular delivery mechanism of CBSA-PO in vitro. ${ }^{34,35}$ Polymersome uptake by bEnd. 3 cells was found to be temperature-dependent, and the uptake index for both CBSA-PO and polymersomes at $37^{\circ} \mathrm{C}$ was much higher than that at $4^{\circ} \mathrm{C}(P<0.01)$. CBSA conjugation with the polymersomes significantly increased the cell uptake by 1.6-fold compared with that of nonconjugated polymersomes at $37^{\circ} \mathrm{C}$ (Figure $3 \mathrm{~A}$ ). The effects of different inhibitors on polymersome uptake are shown in Figure 3B and C. Energy depletion by sodium azide significantly decreased the intracellular uptake of both CBSA-PO and polymersomes, indicating that polymersome uptake was energy-dependent. PheASO and hypertonic sucrose, which are inhibitors of endocytosis, also greatly decreased uptake of polymersomes. In addition, the uptake of CBSAPO was strongly inhibited in the presence of free polycations, such as CBSA and poly-L-lysine, while the uptake of polymersomes was not affected. Furthermore, filipin, which is a specific inhibitor of caveolae-associated endocytosis, significantly decreased the uptake of CBSA-PO but not that of polymersomes. All of these results suggest that the uptake of CBSA-PO was subject to absorptive-mediated endocytosis, and that caveolae were involved in the endocytosis process.

\section{In vitro cytotoxicity of CBSA-PO}

As shown in Figure 4, free CBSA was toxic at high concentrations, while CBSA-PO and polymersomes had little toxicity against bEND. 3 cells. Even at the highest concentration $(8 \mathrm{mg} / \mathrm{mL})$, cell viability for the CBSA-PO group 
A

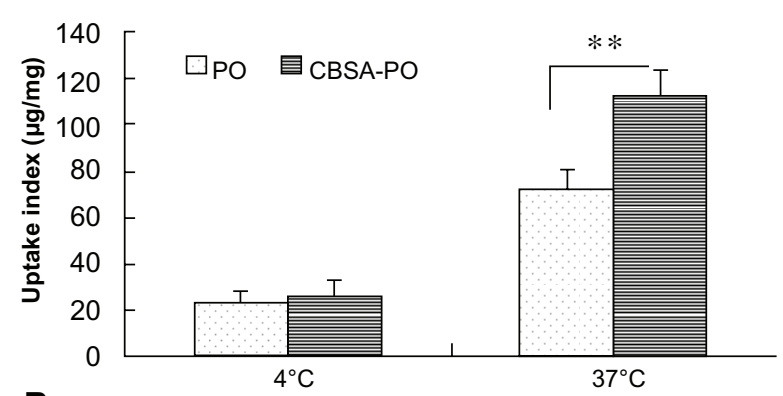

B

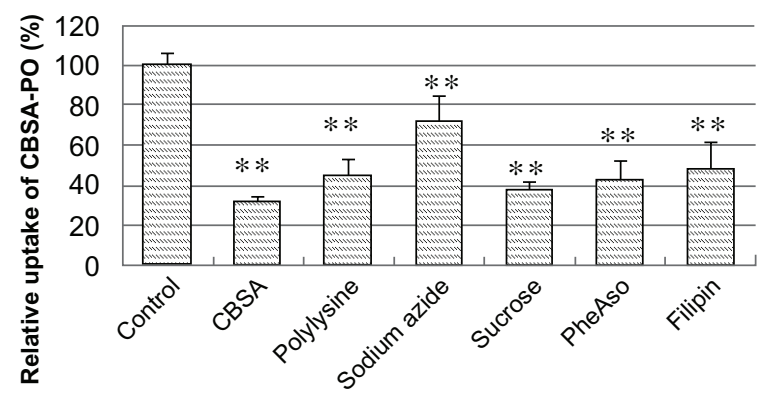

C

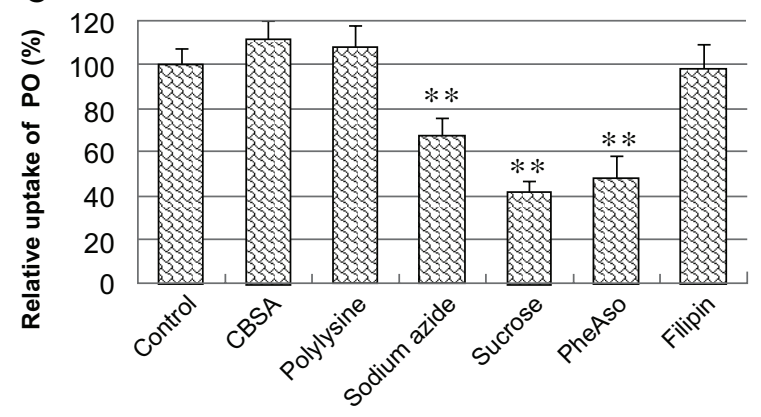

Figure 3 Cell uptake of polymersomes at $(\mathbf{A})$ different incubation temperatures and in the presence of various inhibitors of endocytosis for (B) CBSA-PO and (C) PO. Notes: The bEND. 3 cells were incubated with $100 \mu \mathrm{g} / \mathrm{mL}$ PO or CBSA-PO for one hour. Data presented as the mean \pm standard deviation $(n=5)$. $* * P<0.01$, statistically significant differences by Student's $t$-test when compared with the corresponding value of the control.

Abbreviations: $\mathrm{PO}$, polymersomes; CBSA, cationic bovine serum albumin; pheASO, phenylarsine oxide.

and polymersome group was above $85 \%$, and there were no significant differences in cell viability in comparison with the control. No significant differences in cell viability were observed between the polymersome and CBSA-PO groups. These results confirmed the good safety of biodegradable CBSA-PO. The difference in cell viability between CBSA-PO and CBSA might be related to the CBSA concentrations in the solution. For CBSA-PO, assuming that the mean number of CBSA molecules per polymersome on the surface of CBSA-PO was $95.8 \pm 9.2$, the CBSA content amounted to about $3.5 \%(\mathrm{w} / \mathrm{w})$, which was a very small proportion of the total amount of CBSA-PO. Even

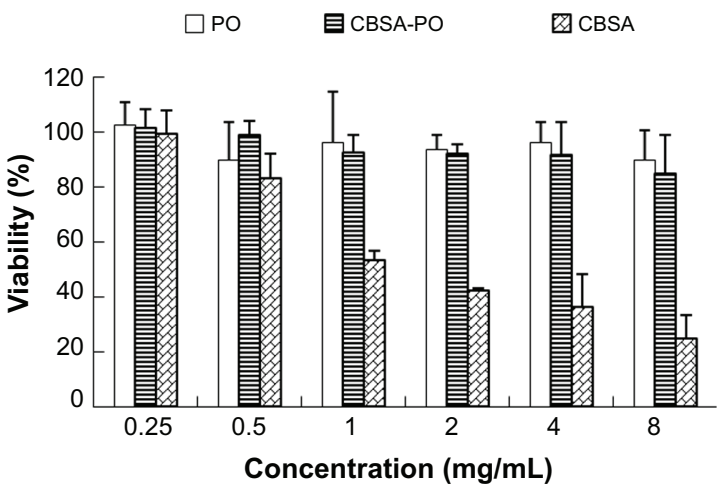

Figure 4 In vitro cytotoxicity of PO, CBSA-PO, and CBSA against bEnd. 3 cells, in a series of concentrations from 0.25 to $8 \mathrm{mg} / \mathrm{mL}$.

Note: Data presented as the mean \pm standard deviation $(n=4)$.

Abbreviations: $\mathrm{PO}$, polymersomes; CBSA, cationic bovine serum albumin.

at the highest concentration $(8 \mathrm{mg} / \mathrm{mL})$ of CBSA-PO, the CBSA concentration in the solution was only $0.28 \mathrm{mg} / \mathrm{mL}$, a low concentration demonstrating little toxicity against bEnd. 3 cells.

\section{Pharmacokinetics and brain delivery of coumarin-6 loaded CBSA-PO}

To investigate delivery of CBSA-PO to the brain, pharmacokinetic experiments were performed in Sprague Dawley rats following intravenous injection of coumarin-6 labeled CBSA-PO at a dosage of $10 \mathrm{mg} / \mathrm{kg}$. Disappearance of CBSAPO from the blood circulation compartment occurred in a biexponential manner (Figure 5). The plasma clearance, area under the plasma concentration curve at steady-state $\left(\mathrm{AUC}_{\mathrm{ss}}\right)$,

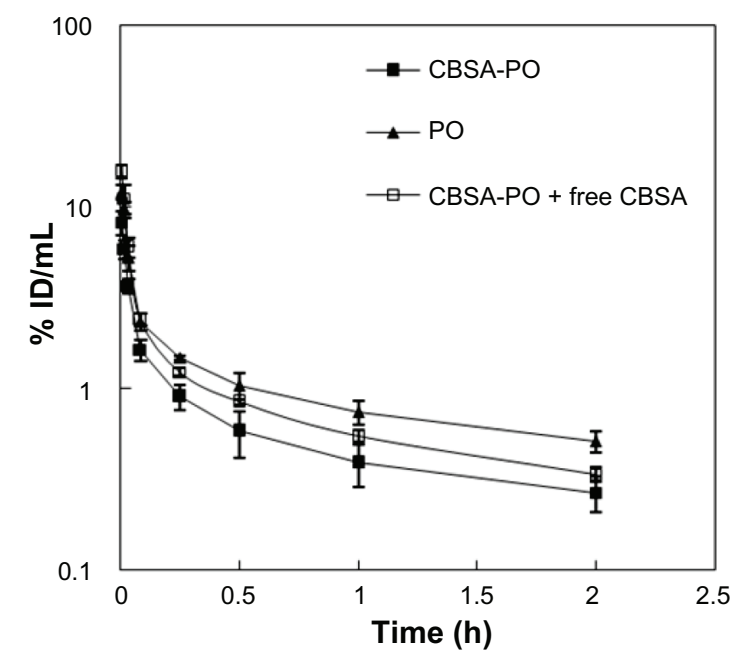

Figure 5 Blood polymersome concentration $(\% \mathrm{ID} / \mathrm{mL})$ versus time after intravenous injection of $10 \mathrm{mg} / \mathrm{kg}$ of PO, CBSA-PO, and CBSA-PO plus $10 \mathrm{mg} / \mathrm{kg}$ of free CBSA. Note: Data are presented as the mean \pm standard deviation of $n=4$ rats/point.

Abbreviations: PO, polymersomes; CBSA, cationic bovine serum albumin; ID, the injected dose. 
and mean residence time were calculated from the data in Figure 5 and are presented in Table 3. All the polymersomes had long circulation times; however, polymersomes conjugated with CBSA showed an increase in plasma clearance and a decrease in mean residence time compared with nonconjugated polymersomes (Figure 5 and Table 3). Although the $\mathrm{AUC}_{0-\mathrm{t}}$ for CBSA-PO was only $59 \%$ that of the polymersomes, the brain permeability surface area product for CBSA-PO was 3.6-fold that of the polymersomes (Figure 6). The average $\%$ ID/g brain tissue for CBSA-PO at 2 hours was $0.11 \%$, which was increased by 2.1 -fold compared with polymersomes, suggesting that CBSA-PO might be a promising carrier for drug delivery to the brain.

\section{Brain delivery kinetics of coumarin-6 loaded CBSA-PO}

Capillary depletion experiments showed that the polymersomes distributed into the rat brain in a time-dependent manner. As shown in Figure 6, the concentration of CBSA-PO in the total brain (the homogenate) that reached the highest value at one hour was significantly higher than that of the polymersomes at $0.5,1,2$, and 4 hours after injection. However, total brain uptake of CBSA-PO was significantly decreased by coinjection of free CBSA. These results are in agreement with those of the pharmacokinetic study. Polymersome concentrations for the CBSA-PO group in the vascular pellet compartment were significantly higher in comparison with those for the polymersome group, but decreased on coinjection of free CBSA (Figure 6B), demonstrating a much higher rate of binding and internalization of CBSA-PO into the brain vasculature in vivo as compared with nonconjugated polymersomes. The polymersome concentration in the postvascular supernatant, which represents the polymersomes entering the brain parenchyma, was very low at all time points, ranging between $0.01 \% \mathrm{ID} / \mathrm{g}$ and $0.04 \% \mathrm{ID} / \mathrm{g}$. Compared with the polymersome group, the polymersome concentration in the CBSA-PO group was much higher, reaching approximately $0.15 \% \mathrm{ID} / \mathrm{g}$ at one hour (Figure $6 \mathrm{C}$ ). The $\mathrm{AUC}_{0-\mathrm{t}}$ in the postvascular supernatant for the CBSA-PO group was 5.3-fold that for the polymersome group, indicating that the polymersomes conjugated with CBSA had significantly improved transcytosis across the blood-brain barrier. As with the total brain uptake of polymersomes, coinjection of free CBSA and CBSA-PO significantly decreased accumulation of CBSA-PO in the brain parenchyma. In addition, the ratio of the CBSAPO concentration in the postvascular supernatant to that of the vascular pellet increased from 0.8 to 2.7 between 0.5 and 4 hours post injection, while that of the polymersomes did not change significantly (Figure 6D), which provides direct evidence of the CBSA-PO transcytosis process across the blood-brain barrier.

\section{Discussion}

This study elucidates the brain delivery kinetics of polymersomes after conjugation with CBSA. It is shown that polymersomes distribute into the rat brain in a timedependent manner and conjugation of polymersomes with CBSA significantly improves their transcytosis across the blood-brain barrier. The polymersome concentrations in the postvascular supernatant (representing the brain parenchyma) in the CBSA-PO group are 2.3-8.8-fold those of the polymersome group after intravenous injection, indicating that the parenchymal concentration of CBSA-PO was higher than that of nonconjugated polymersomes at all time points. In addition, the $\mathrm{AUC}_{0-\mathrm{t}}$ in the postvascular supernatant for the CBSA-PO group was 5.3-fold that of the polymersome group. Moreover, the ratio of the CBSA-PO concentration in the postvascular supernatant to that of the vascular pellet increased dramatically with time, while that of the polymersomes did not change significantly, which provides direct evidence of CBSA-PO transport across the blood-brain barrier. Furthermore, one hour after injection, the CBSA-PO concentration in the postvascular supernatant was approximately $68 \%$ of that in the total homogenate, indicating high efficiency of CBSA-PO transcytosis through the blood-brain barrier.

Table 3 Steady-state area under the plasma concentration-time curve and plasma clearance of different formulations labeled with coumarin-6

\begin{tabular}{llllll}
\hline Group & AUC $_{\text {ss }}(\%$ ID*h/mL) & $\mathbf{C l}(\mathbf{m L} / \mathbf{h} / \mathbf{k g})$ & MRT (hours) & PS ( $\mu$ L/h/g tissue) & $\%$ ID/g tissue \\
\hline PO & $2.94 \pm 0.52$ & $154 \pm 24$ & $1.47 \pm 0.34$ & $23.8 \pm 2.7$ & $0.052 \pm 0.013$ \\
CBSA-PO & $1.37 \pm 0.42^{* *}$ & $343 \pm 83^{* *}$ & $0.82 \pm 0.25^{*}$ & $86.3 \pm 8.6^{* *}$ & $0.111 \pm 0.015^{* *}$ \\
CBSA-PO + CBSA & $2.20 \pm 0.34^{* \dagger}$ & $205 \pm 30^{*, \dagger}$ & $0.96 \pm 0.36$ & $37.5 \pm 5.8^{*, \dagger}$ & $0.075 \pm 0.019^{*, \dagger}$ \\
\hline
\end{tabular}

Notes: Data are the mean \pm standard deviation, $n=4$. Statistically significant differences by Student's $t$-test when compared with the corresponding value of control: $* P<0.05 ; * * P<0.0$ I versus $\mathrm{PO} ; \mathrm{t} P<0.05 ; \forall P<0.0$ I versus CBSA-PO.

Abbreviations: $\mathrm{AUC}_{\text {ss }}$, steady-state area under the concentration time curve; $\mathrm{Cl}$, clearance; $\mathrm{MRT}$, mean residence time; PO, polymersomes; PS, permeability surface area product; CBSA, cationic bovine serum albumin. 
A
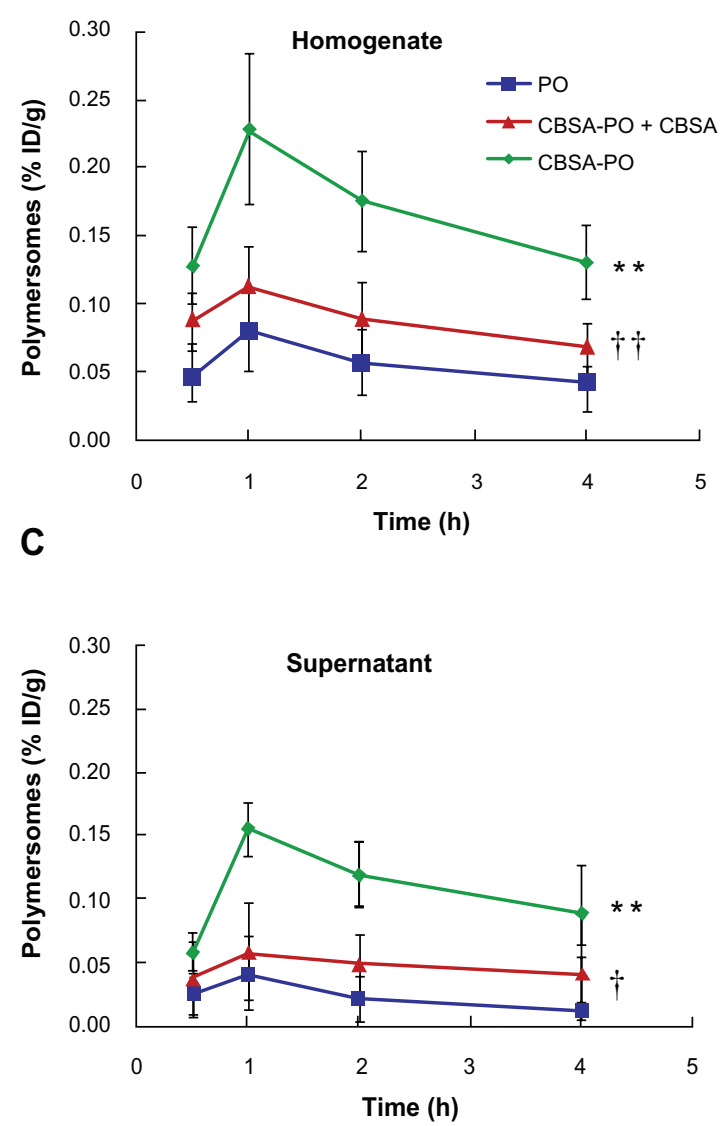

B

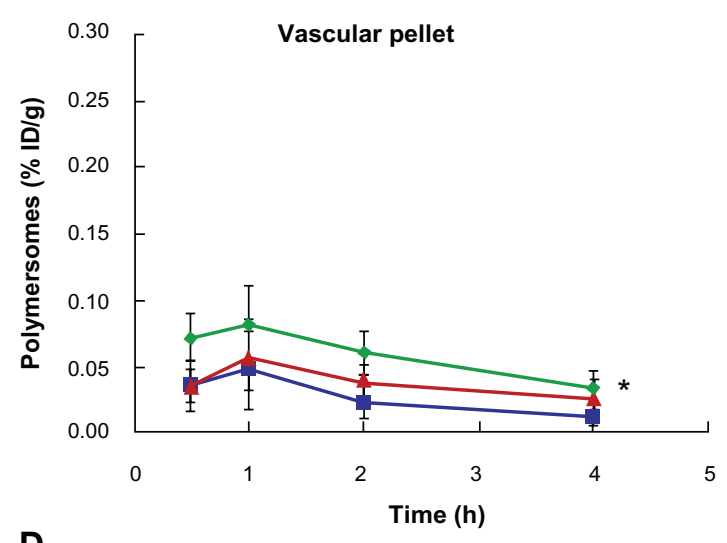

D

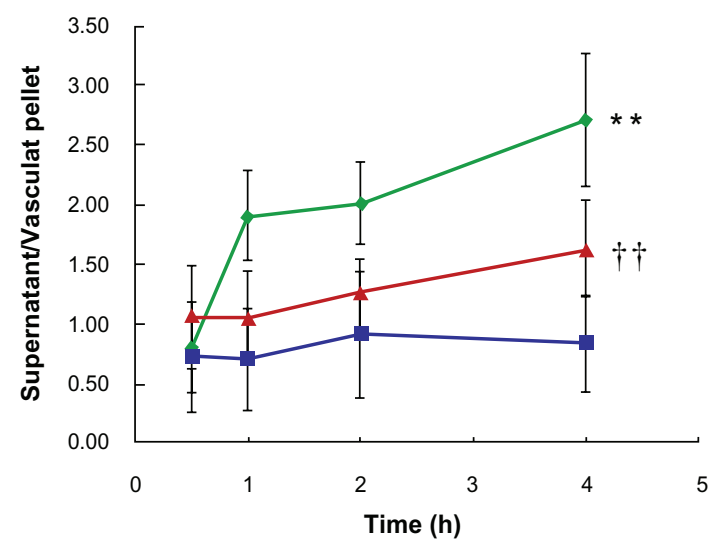

Figure 6 Polymersome concentrations (\% ID/g) versus time curves in the rat brain fractions after intravenous injection of 10 mg/kg of PO, CBSA-PO, and CBSA-PO plus $10 \mathrm{mg} / \mathrm{kg}$ of free CBSA. Data are presented as the mean \pm standard deviation of $n=4$ rats/point. (A) Whole homogenate, (B) vascular pellet, (C) supernatant, and (D) ratio of polymersomes concentrations in the supernatant to those in the vascular pellet.

Notes: Statistically significant differences by Student's $t$-test when compared with the corresponding value of control: $* P<0.05$; $* * P<0.01$ versus $P O$; ${ }^{\dagger} P<0.05$; ${ }^{\dagger} P<0.0$ I versus CBSA-PO.

Abbreviations: $\mathrm{PO}$, polymersomes; CBSA, cationic bovine serum albumin.

In this study, CBSA-PO proved to be an efficient brain drug delivery system in vitro and in vivo, which might be attributable to the optimal chemical and physical properties of the polymersomes, including their size, surface charge, and surface chemistry. Moderate CBSA density on the polymersomes improved the blood-brain barrier permeability

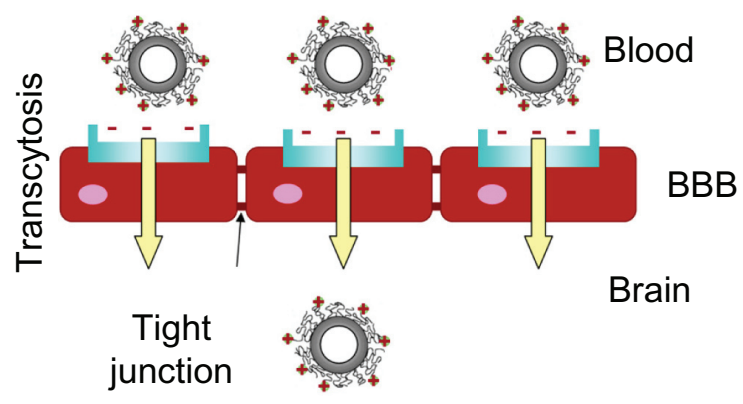

Graphic abstract Brain delivery kinetics of cationic bovine serum albuminconjugated biodegradable polymersomes. Abbreviation: BBB, blood-brain barrier. to polymersomes, but showed moderate blood clearance. In a previous report, we showed that a higher density of CBSA on PEGylated nanoparticles increased not only blood-brain barrier permeability to nanoparticles but also clearance of these nanoparticles from the blood, and that a moderate CBSA density on nanoparticles (109.9 per nanoparticle) was optimal for brain delivery ${ }^{31}$ In the present study, the mean number of CBSA molecules per polymersome on the surface of CBSA-PO was $95.8 \pm 9.2$, which is close to the optimal CBSA density on nanocarriers. Our in vitro study showed that uptake of polymersomes by bEnd. 3 cells was significantly increased (by 1.6-fold) after surface conjugation with CBSA. Further in vivo biodistribution experiments demonstrated that use of CBSA-conjugated polymersomes significantly increased the brain permeability surface area value (by 3.6fold) while the AUC only decreased to $59 \%$. According to the equation, \% ID/gt $(t)=P S \times A U C_{0-t}$, uptake of CBSA-PO by the brain at 2 hours increased by 2.1 -fold that of the uptake 
for polymersomes. A high PEG density on the surface of the polymersomes afforded good resistance to opsonization and extended vesicle circulation times. ${ }^{12} \mathrm{X}$-ray photoelectron spectroscopy confirmed that a corona layer with a high PEG density (30.8\%) existed on the polymersome surface, which was higher than for PEGylated nanoparticles (21.7\%). ${ }^{27}$ In vivo biodistribution studies demonstrated that biodegradable polymersomes had longer circulation times compared with PEGylated nanoparticles. ${ }^{31}$ Although CBSA conjugation increased the zeta potential of polymersomes from $-20 \mathrm{mV}$ to around $+9 \mathrm{mV}$, which might increase clearance of polymersomes from the blood, ${ }^{36}$ this value still agrees well (within $10 \mathrm{mV}$ ) with the optimal zeta potential for in vivo delivery of nanocarriers. ${ }^{37}$ Finally, maintaining a vesicle size around $100 \mathrm{~nm}$ might be optimal for improving the pharmacokinetics of polymersomes ${ }^{38}$ and advantageous for endocytosis by brain capillary endothelial cells. ${ }^{30,39}$

There are three negatively charged barriers at the bloodbrain barrier. ${ }^{40}$ At systemic physiological $\mathrm{pH}$, the luminal and abluminal surfaces of brain capillary endothelial cells have an overall negative charge, and the basement membrane contiguous to the basolateral side of the brain capillary endothelial cells is also a negatively charged barrier, due to its chondroitin-rich and heparin sulfate-rich glycosaminoglycans components. ${ }^{40}$ All three of these successive electrostatic barriers create an environment for selective transport of cationic substances from the blood to the brain. The luminal side provides anionic sites for interacting with cationic substances and inducing absorptive-mediated endocytosis. The anionic properties of both the abluminal cell side and the basement membrane can facilitate externalization of cationic substances from the lumen to the brain interstitium. ${ }^{40}$ It has been shown that cationic albumin not only binds to the surfaces of brain capillary endothelial cells but also increases the uptake of proteins and nanoparticles conjugated with cationic albumin by the brain via absorptive-mediated transcytosis. ${ }^{20,22}$ In the present study, CBSA was conjugated with biodegradable polymersomes, and the brain uptake mechanism of CBSA-PO was investigated. Similar to the brain delivery of other cationic substances via absorptivemediated transcytosis, the cellular uptake of CBSA-PO was energy-dependent because energy depletion and/or a low temperature greatly reduced uptake of polymersomes and CBSA-PO by the cells. Inhibitors of endocytosis, such as PheASO and hypertonic sucrose, also greatly decreased the uptake of polymersomes, indicating that the polymersomes were internalized by the cells via endocytosis. In addition, free polycations, such as CBSA and poly-L-lysine, strongly inhibited cellular uptake of CBSA-PO but not of polymersomes. Further in vivo brain delivery results revealed that coadministration of CBSA and CBSA-PO significantly decreased the blood-brain barrier permeability to CBSA-PO as well as its uptake by the brain. These results suggested that uptake of CBSA-PO by the brain was subject to absorptivemediated transcytosis. Clathrin-coated pits or caveolae might be involved in transcytosis at the blood-brain barrier. The uptake of Syn-B peptides, a family of cationic cell-penetrating peptides, starts with the caveolae-independent pathway. ${ }^{41}$ For natural polyamines, two endocytic pathways have been described in various cultured cells. ${ }^{42,43}$ In this study, filipin, which is a specific inhibitor of caveolae-associated endocytosis, significantly decreased the uptake of CBSAPO but not of polymersomes, suggesting that caveolae are involved in the endocytosis of CBSA-PO. However, whether clathrin-coated pits are involved in transcytosis and which anion component at the blood-brain barrier plays a critical role in cellular internalization of CBSA-PO are still in need of verification and more pharmacological inhibitors might need to be used to elucidate the endocytosis mechanism of polymersomes more clearly.

\section{Conclusion}

A novel brain drug delivery system, based on biodegradable PEG-PCL polymersomes conjugated with CBSA, was established for the first time to the authors' knowledge. Coupling of CBSA with polymersomes was confirmed by X-ray photoelectron spectroscopy. It was shown that uptake of CBSA-PO by bEnd.3 cells was energy-driven and absorptive-mediated. Pharmacokinetic and brain distribution results demonstrated that polymersomes had long circulation times, and CBSA conjugation on polymersomes significantly increased the blood-brain barrier permeability surface area product and percentage of injected dose per gram of brain. Moreover, capillary depletion experiments showed that CBSA-PO distributed to the brain parenchyma in a time-dependent manner and CBSA conjugation with polymersomes significantly improved their transcytosis across the blood-brain barrier. Cell viability assays confirmed good safety of CBSA-PO for delivery to the brain. These results suggest that CBSA-PO is a promising brain delivery carrier with low toxicity.

\section{Acknowledgments}

This work was supported by the National Basic Research Program of China (973 Program, 2007CB935800), National Science and Technology Major Project 
(2009ZX09310-006), National Natural Science Foundation of China (81001404, 81100345), and the Doctoral fund of Ministry of Education of China (20100071120050). Thanks are extended to Professor Weilin Dai, Division of Physics, Fudan University, China, for his advice and help with the $\mathrm{X}$-ray photoelectron spectroscopy analysis.

\section{Disclosure}

The authors report no conflicts of interest in this work.

\section{References}

1. Pardridge WM. Blood-brain barrier delivery. Drug Discov Today. 2007;12: 54-61.

2. Pardridge WM. Drug and gene targeting to the brain with molecular Trojan horses. Nat Rev Drug Discov. 2002;1:131-139.

3. Wong HL, Wu XY, Bendayan R. Nanotechnological advances for the delivery of CNS therapeutics. Adv Drug Deliv Rev. 2012;64: 686-700.

4. Ke W, Shao K, Huang R, et al. Gene delivery targeted to the brain using an Angiopep-conjugated polyethyleneglycol-modified polyamidoamine dendrimer. Biomaterials. 2009;30:6976-6985.

5. Gan CW, Feng SS. Transferrin-conjugated nanoparticles of poly(lactide)-D-alpha-tocopheryl polyethylene glycol succinate diblock copolymer for targeted drug delivery across the blood-brain barrier. Biomaterials. 2010;31:7748-7757.

6. Kreuter J, Hekmatara T, Dreis S, Vogel T, Gelperina S, Langer K. Covalent attachment of apolipoprotein A-I and apolipoprotein B-100 to albumin nanoparticles enables drug transport into the brain. J Control Release. 2007;118:54-58.

7. Liu Y, Huang R, Han L, et al. Brain-targeting gene delivery and cellular internalization mechanisms for modified rabies virus glycoprotein RVG29 nanoparticles. Biomaterials. 2009;30:4195-4202.

8. Li J, Feng L, Fan L, et al. Targeting the brain with PEG-PLGA nanoparticles modified with phage-displayed peptides. Biomaterials. 2011;32: 4943-4950.

9. Chen Q, Schonherr H, Vancso GJ. Block-copolymer vesicles as nanoreactors for enzymatic reactions. Small. 2009;5:1436-1445.

10. Christian DA, Cai S, Bowen DM, Kim Y, Pajerowski D, Discher DE. Polymersome carriers: from self-assembly to siRNA and protein therapeutics. Eur J Pharm Biopharm. 2009;71:463-474.

11. Meng F, Engbers GH, Feijen J. Biodegradable polymersomes as a basis for artificial cells: encapsulation, release and targeting. J Control Release. 2005;101:187-198.

12. Discher DE, Ortiz V, Srinivas G, et al. Emerging applications of polymersomes in delivery: from molecular dynamics to shrinkage of tumors. Prog Polym Sci. 2007;32:838-857.

13. Discher DE, Eisenberg A. Polymer vesicles. Science. 2002;297: 967-973.

14. Du J, O'Reilly RK. Advances and challenges in smart and functional polymer vesicles. Soft Matter. 2009;5:3544-3561.

15. Meng F, Hiemstra C, Engbers GHM, Feijen J. Biodegradable polymersomes. Macromolecules. 2003;36:3004-3006.

16. Christian NA, Milone MC, Ranka SS, et al. Tat-functionalized nearinfrared emissive polymersomes for dendritic cell labeling. Bioconjug Chem. 2007;18:31-40.

17. Meng F, Zhong Z, Feijen J. Stimuli-responsive polymersomes for programmed drug delivery. Biomacromolecules. 2009;10: 197-209.

18. Pang Z, Lu W, Gao H, et al. Preparation and brain delivery property of biodegradable polymersomes conjugated with OX26. J Control Release. 2008;128:120-127.

19. Gaillard PJ, Visser CC, Boer AG. Targeted delivery across the bloodbrain barrier. Expert Opin Drug Deliv. 2005;2:299-309.
20. Kumagai AK, Eisenberg JB, Pardridge WM. Absorptive-mediated endocytosis of cationized albumin and a beta-endorphin-cationized albumin chimeric peptide by isolated brain capillaries. Model system of blood-brain barrier transport. J Biol Chem. 1987;262:15214-15219.

21. Bickel U, Yoshikawa T, Pardridge WM. Delivery of peptides and proteins through the blood-brain barrier. Adv Drug Deliv Rev. 2001;46: 247-279.

22. Lu W, Sun Q, Wan J, She Z, Jiang XG. Cationic albumin-conjugated pegylated nanoparticles allow gene delivery into brain tumors via intravenous administration. Cancer Res. 2006;66:11878-11887.

23. Xie YL, Lu W, Jiang XG. Improvement of cationic albumin conjugated pegylated nanoparticles holding NC-1900, a vasopressin fragment analog, in memory deficits induced by scopolamine in mice. Behav Brain Res. 2006;173:76-84.

24. Yoshikawa T, Pardridge WM. Biotin delivery to brain with a covalent conjugate of avidin and a monoclonal antibody to the transferrin receptor. J Pharmacol Exp Ther. 1992;263:897-903.

25. Wohlfart S, Khalansky AS, Gelperina S, Begley D, Kreuter J. Kinetics of transport of doxorubicin bound to nanoparticles across the bloodbrain barrier. J Control Release. 2011;154:103-107.

26. Xu F, Lu W, Wu H, Fan L, Gao X, Jiang X. Brain delivery and systemic effect of cationic albumin conjugated PLGA nanoparticles. J Drug Target. 2009;17:423-434.

27. Lu W, Zhang Y, Tan YZ, Hu KL, Xiang XG, Fu SK. Cationic albuminconjugated pegylated nanoparticles as novel drug carrier for brain delivery. J Control Release. 2005;107:428-448.

28. Lu W, Tan YZ, Hu KL, Jiang XG. Cationic albumin conjugated pegylated nanoparticle with its transcytosis ability and little toxicity against blood-brain barrier. Int J Pharm. 2005;295:247-260.

29. Kang YS, Bickel U, Pardridge WM. Pharmacokinetics and saturable blood-brain barrier transport of biotin bound to a conjugate of avidin and a monoclonal antibody to the transferrin receptor. Drug Metab Dispos. 1994;22:99-105.

30. Huwyler J, Wu D, Pardridge WM. Brain drug delivery of small molecules using immunoliposomes. Proc Natl Acad Sci U S A. 1996;93: 14164-14169.

31. Lu W, Wan J, She Z, Jiang X. Brain delivery property and accelerated blood clearance of cationic albumin conjugated pegylated nanoparticle. J Control Release. 2007;118:38-53.

32. Peracchia MT, Gref R, Minamitake Y, Domb A, Lotan N, Langer R. PEG-coated nanospheres from amphiphilic diblock and multiblock copolymers: investigations of their drug encapsulation and release characteristics. J Control Release. 1997;46:223-231.

33. Davda J, Labhasetwar V. Characterization of nanoparticle uptake by endothelial cells. Int J Pharm. 2002;233:51-59.

34. Brown RC, Morris AP, O’Neil RG. Tight junction protein expression and barrier properties of immortalized mouse brain microvessel endothelial cells. Brain Res. 2007;1130:17-30.

35. Hu K, Li J, Shen Y, et al. Lactoferrin-conjugated PEG-PLA nanoparticles with improved brain delivery: in vitro and in vivo evaluations. J Control Release. 2009;134:55-61.

36. He C, Hu Y, Yin L, Tang C, Yin C. Effects of particle size and surface charge on cellular uptake and biodistribution of polymeric nanoparticles. Biomaterials. 2010;31:3657-3666.

37. Li SD, Huang L. Pharmacokinetics and biodistribution of nanoparticles. Mol Pharm. 2008;5:496-504.

38. Alexis F, Pridgen E, Molnar LK, Farokhzad OC. Factors affecting the clearance and biodistribution of polymeric nanoparticles. Mol Pharm. 2008;5:505-515.

39. Massignani M, LoPresti C, Blanazs A, et al. Controlling cellular uptake by surface chemistry, size, and surface topology at the nanoscale. Small. 2009;5:2424-2432.

40. Herve F, Ghinea N, Scherrmann JM. CNS delivery via adsorptive transcytosis. AAPS J. 2008;10:455-472.

41. Drin G, Cottin S, Blanc E, Rees AR, Temsamani J. Studies on the internalization mechanism of cationic cell-penetrating peptides. $J$ Biol Chem. 2003;278:31192-311201. 
42. Belting M, Mani K, Jonsson M, et al. Glypican-1 is a vehicle for polyamine uptake in mammalian cells. A pivotal role for nitrosothiolderived nitric oxide. J Biol Chem. 2003;278:47181-47189.
43. Soulet D, Gagnon B, Rivest S, Audette M, Poulin R. A fluorescent probe of polyamine transport accumulates into intracellular acidic vesicles via a two-step mechanism. J Biol Chem. 2004;279:49355-49366.

\section{Publish your work in this journal}

The International Journal of Nanomedicine is an international, peerreviewed journal focusing on the application of nanotechnology in diagnostics, therapeutics, and drug delivery systems throughout the biomedical field. This journal is indexed on PubMed Central, MedLine, CAS, SciSearch ${ }^{\circledR}$, Current Contents ${ }^{\circledR} /$ Clinical Medicine,
Journal Citation Reports/Science Edition, EMBase, Scopus and the Elsevier Bibliographic databases. The manuscript management system is completely online and includes a very quick and fair peer-review system, which is all easy to use. Visit http://www.dovepress.com/ testimonials.php to read real quotes from published authors. 